\title{
Publisher Correction: Bosonic condensation of exciton-polaritons in an atomically thin crystal
}

Carlos Anton-Solanas (D), Maximilian Waldherr, Martin Klaas, Holger Suchomel, Tristan H. Harder @ , Hui Cai,

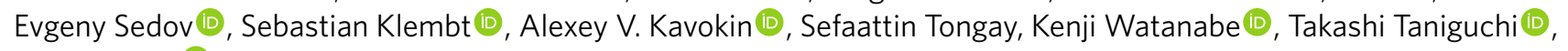
Sven Höfling (1) and Christian Schneider

Correction to: Nature Materials https://doi.org/10.1038/s41563-021-01000-8, published online 6 May 2021.

In the version of this Article originally published, the affiliation for the author Christian Schneider was mistakenly given as 'Technische Physik and Würzburg-Dresden Cluster of Excellence ct.qmat, Universität Würzburg, Würzburg, Germany', but it should have been 'Institute of Physics, Carl von Ossietzky University, Oldenburg, Germany'. This has now been corrected.

Published online: 14 May 2021

https://doi.org/10.1038/s41563-021-01036-w

(c) The Author(s), under exclusive licence to Springer Nature Limited 2021

\section{Publisher Correction: Synthetic Rashba spin-orbit system using a silicon metal-oxide semiconductor}

Soobeom Lee, Hayato Koike, Minori Goto, Shinji Miwa B, Yoshishige Suzuki, Naoto Yamashita, Ryo Ohshima (D, Ei Shigematsu, Yuichiro Ando and Masashi Shiraishi (D)

Correction to: Nature Materials https://doi.org/10.1038/s41563-021-01026-y, published online 3 June 2021.

In the version of this Article originally published, the units of the Rashba parameter were incorrect as ' $\mathrm{eV} \mathrm{m}^{-1}$ ' in the abstract, in the section 'Physics behind of the spin lifetime anisotropy', and in the $y$-axis label of Fig. 4c; the units should have been ' $\mathrm{eV}$ m'. This has now been corrected.

Published online: 28 July 2021

https://doi.org/10.1038/s41563-021-01086-0

(C) The Author(s), under exclusive licence to Springer Nature Limited 2021 